\title{
Skin Lymphangioleiomyomatosis
}

National Cancer Institute

\section{Source}

National Cancer Institute. Skin Lymphangioleiomyomatosis. NCI Thesaurus. Code C4370.

Lymphangiomyomatosis involving the dermis. 